\title{
Imunologia da Doença por Coronavírus-19 (COVID-19): Uma Perspetiva Para o Clínico, nos Primeiros 4 Meses da Emergência do SARS-COV-2
}

\author{
Immunology of Coronavirus-19 Disease (COVID-19): A Perspective for \\ the Clinician in the First 4 Months of the Emergence of SARS-CoV-2
}

\author{
José Laerte Boechat ${ }^{1,2}$ (https://orcid.org/ 0000-0002-9738-8210), Inês Chora ${ }^{3,4}$ (https://orcid.org/ 0000-0003-4750-2326), \\ Luís Delgado ${ }^{2,5}$ (https://orcid.org/ 0000-0003-2375-907)
}

\section{Resumo:}

Nos quatro meses desde a emergência duma infeção pandémica por uma nova estirpe altamente patogénica de coronavírus beta em humanos, a COVID-19, assistimos a uma rápida partilha nas plataformas digitais das mais diversas publicações científicas, numa experiência que se vive também pela primeira vez na comunidade internacional. Neste trabalho, fazemos uma revisão descritiva das mais recentes informações sobre a imunobiologia do SARS-CoV-2, focando as hipóteses que suportam uma deficiência nos mecanismos da imunidade inata e adquirida ao vírus, e o contributo de uma resposta imunitária disfuncional na progressão e mortalidade associadas à doença. Com base nessa nova informação, apontamos as principais estratégias imunológicas nas terapêuticas dirigidas ao vírus, à resposta imune disfuncional e à profilaxia da infeção (imunização). O aprofundar do conhecimento sobre as interações patogénio-hospedeiro, abrirá o caminho para identificar as melhores estratégias para o diagnóstico, terapêutica e controlo da COVID-19, bem como para a sua prevenção.

Palavras-chave: Coronavírus; Infecções por Coronavírus; Imunopatologia; Pandemia.

\section{Abstract:}

In the last four months, since the emergence of a pandemic infection by a new highly pathogenic strain of beta coronavirus in humans, COVID-19, we have witnessed the fast sharing on digital platforms of the most diverse scientific publications, an experience also lived for the first time by the international

${ }^{1}$ Serviço de Imunologia Clínica, Departamento de Clínica Médica, Faculdade de Medicina, Universidade Federal Fluminense, Niterói/ RJ, Brasil.

'Serviço de Imunologia Básica e Clínica, Departamento de

Patologia, Faculdade de Medicina, Universidade do Porto, Portugal. ${ }^{3}$ Serviço de Medicina Interna, Departamento de Medicina, Hospita Pedro Hispano, Unidade Local de Saúde de Matosinhos, Senhora da Hora, Matosinhos, Portugal

${ }^{4}$ Faculdade de Medicina, Universidade do Porto, Porto, Portugal.

${ }^{5}$ CINTESIS, Centro de Investigação em Tecnologias e Serviços de

Saúde, Universidade do Porto, Porto, Portugal.

https://revista.spmi.pt - DOI: 10.24950/rspmi /COVID19/FMUP/S/2020 medical community. In this review of the latest information on SARS-COV-2 immunobiology, we focused on the hypotheses that support a defective innate and acquired immunity to the virus, and the contribution of a dysfunctional immune response for the progression and mortality associated with the disease. Based on this new information, we point out the main immunotherapy strategies targeting the virus, the dysfunctional immune response and the disease prophylaxis (immunization). Improving our knowledge on the pathogen-host interactions will open the way to identify the best strategies for the diagnosis, treatment and control of COVID-19, as well as to the best preventive measures.

Keywords: Coronavirus; Coronavirus Infections; COVID19; Immunopathology; Pandemics.

\section{Introdução}

Os coronavírus (CoV) são um grupo diverso de vírus de ARN de cadeia simples, que infetam uma diversidade de vertebrados, inicialmente identificados em humanos nos anos $60,{ }^{1}$ sendo essencialmente causadores de doença ligeira das vias respiratórias superiores. No entanto, desde o início deste século surgiram, por transmissão zoonótica, novas infeções por estirpes altamente patogénicas de coronavírus beta em humanos; em 2002, a primeira síndrome respiratória aguda severa (SARS-CoV-1) e, em 2012, a síndrome respiratória do médio oriente relacionada com coronavírus (MERS-CoV), ambas com mortalidade elevada por causa respiratória, entre os 10 e 35\% respetivamente. O SARS-CoV-2 é um coronavírus beta semelhante ao SARS-CoV-1, que emergiu na província de Hubei na China nos finais de 2019,2 e a causa da atual doença por coronavírus 2019 (COVID-19).

A 11 de março de 2020, a infeção pelo SARS-CoV-2 já tinha atingido mais de 100 mil pessoas, acima de 4 mil casos letais, em mais de 100 países, e a Organização Mundial de Saúde declarou a situação como pandemia, a primeira originada por um coronavírus na humanidade. ${ }^{3}$ Desde essa data um número exponencial de casos surgiu em múltiplos países e continentes, em grande parte associado à extensa globalização e rápidas deslocações de que muitos usufruímos desde o início deste 
milénio. Em todo o caso, as possibilidades de controlar rapidamente esta pandemia têm sido limitadas por desconhecimento de múltiplos aspetos relevantes do SARS-CoV-2, desde a sua biologia, resposta do hospedeiro (resposta imunológica), ao rápido diagnóstico e identificação de casos até à inexistência de um tratamento eficaz, a esta data. Daí a urgência em aprofundar o conhecimento sobre as interações patogénio-hospedeiro, que abrirão o caminho para identificar as estratégias mais adequadas no diagnóstico, controlo e terapêutica da COVID-19 e na prevenção da disseminação desta nova infeção.

Paralelamente, o conhecimento e informação gerada pela comunidade médica e científica tem progredido de uma forma vertiginosa nos 4 meses desde a emergência desta nova infeção na China, com uma rápida partilha nas plataformas das mais diversas publicações científicas, numa experiência que se vive também pela primeira vez na comunidade internacional. ${ }^{4}$

Neste trabalho fazemos a revisão das publicações mais recentes sobre a imunobiologia do SARS-CoV-2, nas suas interações com as células alvo e a resposta imunitária do hospedeiro, focando particularmente atenção na informação e hipóteses que suportam uma deficiência nos mecanismos da imunidade inata e adquirida ao vírus e no contributo de uma resposta imunitária disfuncional na progressão e mortalidade associada à doença. Abordaremos, também, brevemente, as estratégias de base imunológica para intervenções terapêuticas dirigidas ao vírus, à resposta imune disfuncional e a profilaxia da infeção (imunização).

\section{A resposta Imune ao SARS-CoV-2}

\section{HISTÓRIA NATURAL DA COVID-19}

As consequências clínicas da infeção por SARS-CoV-2 têmse apresentado muito variáveis, desde uma evolução benigna a uma doença rapidamente fatal nas primeiras 2 a 3 semanas após o início dos sintomas. Enquanto muitos indivíduos infetados podem apresentar-se assintomáticos5,6 ou com apenas sintomas respiratórios superiores, outros desenvolvem pneumonia intersticial que pode rapidamente evoluir com insuficiência respiratória e síndrome de dificuldade respiratória aguda (ARDS), necessitando de ventilação mecânica invasiva e admissão em cuidados intensivos (UCl), podendo evoluir com falência

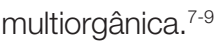

Os casos com evolução mais grave têm uma progressão acelerada. Nas primeiras séries de doentes publicadas, ${ }^{10}$ constata-se uma progressão muito rápida para insuficiência respiratória: o período do início dos sintomas até o aparecimento de ARDS e necessidade de ventilação assistida e admissão em UCI foi em média 1 a 2 semanas - i.e. até à dispneia 8 dias, ARDS 9 dias, UCl/entubação entre 10,5 a 14,5 dias. As principais causas de morte foram a insuficiência respiratória (85\%), um terço dos quais com choque associado. ${ }^{11}$

Tratando-se de um agente patogénico até à data não reconhecido na espécie humana, será sempre de esperar que o desencadear de uma resposta imunológica adaptativa a novos antigénios eficaz na sua neutralização (i.e. uma resposta imune primária de anticorpos e/ou células T) surja no decurso da segunda a terceira semana do contacto. ${ }^{12}$ Esta rápida cronologia clínica parece desde logo apontar para que o controlo da infeção, na maioria assintomática ou com formas ligeiras, se deverá maioritariamente a uma resposta imune do seu componente inato ou inespecífico, i.e. de primeira linha, cuja ativação não depende de um reconhecimento por anticorpos e/ou linfócitos T. Em contraste, as formas de evolução grave poderão associar-se à falha desses mesmos mecanismos de primeira defesa, inespecíficos, e/ou ao emergir de uma resposta imune adquirida, cuja amplificação inflamatória se pode tornar patogénica para o hospedeiro, particularmente quando sofre de comorbilidades importantes.

\section{A DINÂMICA DA RESPOSTA DE ANTICORPOS}

O SARS-CoV-2 é um vírus novo no homem, pelo que anticorpos (atcs) específicos para sua glicoproteína S não são detetáveis na fase inicial da infeção, i.e. antes do estabelecimento de uma resposta imune adaptativa, ${ }^{12}$ tanto mais que atcs para outros coronavírus humanos não reconhecem esses antigénios (atgs). Grande parte dos estudos serológicos disponíveis até esta data referem-se às fases agudas da doença, apontando para o aparecimento de atcs IgM pelo dia 8 a 12, desaparecendo à $12^{\mathrm{a}}$ semana, e dos atcs IgG pelo dia 14, persistindo mais tempo. A intensidade da resposta lgG parece relacionarse com a carga vírica mas, igualmente, com a severidade da doença. ${ }^{13,14} \mathrm{O}$ grau e o tempo de proteção dados por essa resposta são ainda desconhecidos até esta data. Se, por um lado, alguns estudos preliminares sugerem que a transferência passiva de atcs, por plasma de convalescentes, tem eficácia terapêutica, ${ }^{15}$ alguns estudos serológicos recentes revelam haver apenas uma correlação moderada entre a quantidade / título de atcs e a sua atividade de neutralização vírica. ${ }^{16}$ Este último estudo realizado na China, com o plasma de 175 doentes com sintomas moderados e que recuperaram, confirma que na COVID-19 a resposta humoral para o SARS-CoV-2 ocorre entre 10 a 15 dias após a infeção, mas também que cerca de 47\% desses doentes tinham títulos baixos ou muito baixos de atcs neutralizantes, até 2 semanas após a alta hospitalar. Ao discutir como estes doentes recuperam com títulos tão baixos de atcs neutralizantes, os autores sugerem que outros componentes da resposta imune adquirida, que não os atcs, terão contribuído para a recuperação (e.g. a resposta de linfócitos T).

Outros dados recentes, sugerindo que os anticorpos não desempenham um papel crítico na eficácia da resposta imune adquirida é o relato, vindo de Itália, de duas pequenas séries de COVID-19 em doentes com imunodeficiências primárias humorais. ${ }^{17,18}$ Os quatro casos reportados com agamaglobulinemia primária, apresentaram uma evolução favorável, mesmo tendo desenvolvido pneumonia, em comparação com os 5 casos de imunodeficiência comum variável (IDCV), que evoluíram com 
formas mais severas. Apesar de todos os doentes estarem a fazer tratamento com imunoglobulinas endovenosas (IVIG), estas não contêm ainda atcs específicos para o SARS-CoV-2, podendo proteger apenas em relação a infeções secundárias. Não tendo a capacidade de uma resposta de atcs "de novo" eficaz, é de notar que os doentes mantêm o compartimento de imunidade inespecífica intacto (e.g. fagócitos, células NK e interferão). Por outro lado, os autores especulam se as complicações observadas nos doentes com IDCV não poderão estar associadas à existência nestes de células B disfuncionais (ausentes nas agamaglobulinemias) ou de uma resposta de imunidade adquirida de tipo $T$ (celular).

Estas observações parecem suportar a hipótese, recentemente colocada, ${ }^{19}$ de que a imunidade inata será o componente essencial na resposta à infeção vírica na sua fase inicial, antes do aparecimento de um componente da resposta adquirida específica, dependente de linfócitos $B$ e seus atcs e dos linfócitos $T$ e suas citocinas. A falência da imunidade inata abrirá o caminho a uma replicação vírica não controlada nas vias aéreas e o caminho para o emergir de uma resposta adquirida, potencialmente ampliada pela resposta inflamatória.

\section{A RESPOSTA DA IMUNIDADE INATA SARS-COV-2}

A imunidade inata antivírica tem por base diversos componentes humorais, como os dos sistemas do complemento e da coagulação-fibrinólise, proteínas solúveis que reconhecem determinados glicanos na superfície das células (p.ex. a Mannose Binding Lectin que reconhece resíduos de manose), interferões (IFN) e citocinas com ação quimiotática (quimiocinas) e os chamados atcs naturais (lgM, mas também lgA e lgG). Inclui também diversos componentes celulares, entre os quais as células NK, linfócitos inatos (ILCs) e células T gama delta, que geralmente limitam a propagação da infeção vírica por ação citotóxica sobre as células alvo, produção de citocinas e promoção da resposta adaptativa (de linfócitos T e B).

Iremos abordar de seguida alguns desses componentes solúveis, nomeadamente os envolvidos na interação com glicanos da superfície vírica e a produção de interferão após a entrada nas células da mucosa respiratória, mecanismos que têm recebido um interesse crescente na investigação da imunopatogenia da infeção por coronavírus.

O SARS-CoV-2 apresenta uma extensa glicosilação na proteína S (spike) da superfície viral que se liga ao seu recetor nas células do hospedeiro - a enzima de conversão da angiotensina 2 (ACE-2). ${ }^{20}$ A glicosilação da superfície vírica pode influenciar vários aspetos da sua biologia, desde a estabilidade de componentes proteicos, tropismo celular, reconhecimento por mecanismos da imunidade ou, pelo contrário, camuflagem de atgs reconhecidos por atcs neutralizantes.

3.1. Anticorpos naturais - atcs anti-glicanos são detetados no soro naturalmente, i.e. sem imunização prévia, e pertencem essencialmente à classe lgM, como são exemplo os atcs naturais para o sistema $\mathrm{ABO}$ eritrocitário. As concentrações de atcs
IgM naturais parecem espelhar alguns dos padrões associados à maior gravidade da COVID-1919: diminuem significativamente com a idade (a partir dos 40 anos), são em concentração mais baixa nos homens e nos indivíduos do serogrupo sanguíneo $A$. Um papel protetor de títulos elevados de anticorpos anti-A foi descrito para o SARS-CoV-121 e tem sido sugerida também para o SARS-CoV-2.22

3.2 MBL (Mannose Binding Lectin) - a MBL é um dos componentes da imunidade inata reconhecendo resíduos de manose na membrana de uma diversidade de microrganismos, atuando com um recetor de reconhecimento de padrões (PRR), na forma solúvel. Através desse reconhecimento, ativa o sistema do Complemento, induzindo inflamação e potenciando a fagocitose. Sabe-se que a MBL pode ligar o SARS-CoV, levando à deposição de $\mathrm{C} 4$ no vírus e, em modelos experimentais, reduzindo a sua capacidade de infeção. ${ }^{23} \mathrm{~A}$ presença de glicanos ricos em manose na região S1 do SARS-CoV-2, junto do local de ligação ao seu recetor celular ACE-2, tem levantado a hipótese de que o seu reconhecimento e ligação à $\mathrm{MBL}$ poderão inibir a interação S1-ACE. ${ }^{20}$ Por outro lado, alguns polimorfismos genéticos da MBL, que se associam a níveis séricos mais baixos de MBL, foram também associados à suscetibilidade ao SARS-CoV. ${ }^{24}$ Os níveis séricos de MBL parecem também diminuir com a idade. ${ }^{25}$

3.3. Interferões e outras citocinas na resposta à infeção vírica - para muitos vírus respiratórios, incluindo o SARS-CoV-1, os IFN tipo I e III (ditos "inatos"), parecem desempenhar um papel relevante na limitação da infeção. ${ }^{26} A$ doença respiratória aguda provocada pela infeção por SARS-CoV-1 e MERS-CoV tem sido caracterizada por uma resposta inflamatória desregulada, na qual uma produção tardia de IFN tipo I promove a acumulação de monócitos-macrófagos inflamatórios. ${ }^{27}$ Dados recentes ${ }^{28,29}$ apontam também para uma possível desregulação dos IFN tipo I e III na resposta aos SARS-CoV-2.

Os IFN de tipo I (IFN- $\alpha / \beta)$ são dos primeiros mecanismos da defesa inata antivírica, induzindo resistência vírica, quer na célula infetada (efeito autócrino) quer nas células vizinhas (efeito parácrino), "interferindo" com a replicação vírica e celular. Em paralelo, potenciam a resposta imune celular, inata e adaptativa, para eliminação das células infetadas. Uma outra família de IFN da imunidade inata, lambda (IFN- $\lambda$ ), IFN tipo III ou IL-28/ IL-29, partilha homologia com o IFN tipo I e a IL-10. Como os IFN tipo I, são produzidos transitoriamente nas células após interação de derivados microbianos com os seus recetores de reconhecimento de padrões (PRRs), ativando uma cascata de sinalização intracelular (em parte partilhada com os IFN tipo I), transcrição e ativação do fator nuclear kB (NF-kB) e fatores reguladores dos interferões (IRFs). Da ativação dos IRFs e NF-kB resultam duas vias de defesa: a resistência antivírica celular e uma via de recrutamento celular, por produção de diversas quimiocinas. A estas eficazes defesas antivíricas opõem-se mecanismos de escape vírico, ao adquirirem características genéticas de resistência (revisto $\left.\mathrm{em}^{29,30}\right)$. 
Os IFN tipo III têm uma expressão mais restrita do que os do tipo I (ubiquitários), sendo sobretudo das barreiras mucosas (respiratória e gastrointestinal). A principal diferença entre eles é na expressão dos seus recetores: os do IFN tipo I estão em praticamente todas as células e o recetor do tipo III (complexo IFNLR1/IL10RB) em células epiteliais e neutrófilos, assim como em algumas células imunitárias ativadas (células dendríticas, macrófagos e linfócitos B). Estes dados sugerem um papel mais especializado do tipo III na resposta imune das mucosas e na regulação da resposta adaptativa. Os IFN tipo III têm um papel particular na resposta antivírica pulmonar: são induzidos primeiro e por uma carga vírica menor (que o tipo I), limitam a disseminação vírica inicial ${ }^{31}$ e a transmissão vírica do aparelho respiratório superior para o pulmão. ${ }^{32}$ Não têm o potencial próinflamatório dos de tipo I e, pelo contrário, podem ter efeitos anti-inflamatórios e protetores tecidulares (em parte partilhados com a IL-10). ${ }^{26,33}$

Estudos muito recentes, em pré-publicação, de modelos animais da infeção por SARS-CoV-227 sugerem que estas duas famílias de IFNs contribuem para a limitação local (IFN tipo III) e sistémica (IFN tipo I) da disseminação vírica mas, por outro lado, com a indução de uma intensa resposta inflamatória do pulmão e sistémica, pelo IFN tipo I. Outro estudo muito recente, avaliou a resposta transcricional ao SARS-CoV-2, em diversas linhas celulares pulmonares, modelos animais e ex-vivo (tecido pulmonar de doentes com COVID-19) comparativamente com outros vírus respiratórios (Influenza A e SARS-CoV-1) que se sabe codificarem antagonistas da resposta celular ao IFN I/III. ${ }^{28}$ Os resultados apontam para uma desregulação da resposta do hospedeiro, já que o SARS-CoV-2 não desencadeia uma resposta IFN I/III robusta (pelo menos em cargas víricas mais baixas) mas induz produção robusta de quimiocinas, com capacidade de recrutar células inflamatórias. Nestes modelos experimentais, os autores demonstraram uma indução significativa de quimiocinas associadas aos monócitos (CCL2 e CCL8) e de neutrófilos (CXCL2 e CXCL8). Estas observações experimentais são consistentes com dados obtidos em doentes com COVID-19, que têm revelado uma neutrofilia periférica, fator de pior prognóstico, ${ }^{34,35}$ e o predomínio no pulmão de macrófagos derivados da periferia (monócitos), nos casos mais severos. ${ }^{36}$

\section{A imunopatologia da COVID-19}

A fisiopatologia do envolvimento pulmonar por este novo coronavírus assemelha-se muito à infeção por SARS- e MERS$\mathrm{CoV}$, em que uma resposta inflamatória particularmente agressiva tem sido implicada na lesão pulmonar. ${ }^{37,38} \mathrm{~A}$ base patológica desta morbilidade parece ser a lesão das células pulmonares infetadas - os pneumócitos de tipo $\|$ e as células endoteliais capilares - o que leva a compromisso das trocas gasosas pulmonares (hipoxemia) e a um importante exsudado de componentes plasmáticos para o espaço alveolar. A perda da integridade da barreira epitélio-endotelial alveolar é agravada por infiltração de células inflamatórias no interstício e espaço alveolar. Os primeiros estudos histopatológicos confirmaram uma lesão alveolar difusa, com a formação de membranas hialinas, infiltração mononuclear e macrofágica dos espaços aéreos e espessamento difuso da parede alveolar. ${ }^{38}$

Assim, a severidade da COVID-19 parece dever-se não só à infeção mas também à resposta imune e inflamatória do hospedeiro, podendo esta ter por base uma desregulação da resposta imunológica. Dados recentes sugerem também que esta desregulação imune poderá estar implicada num estádio de imunossupressão, ${ }^{39,40}$ que se segue à fase pró-inflamatória (síndrome de "tempestade" de citocinas) e que, clinicamente, se associa a linfopenia periférica e a um risco elevado de infeções bacterianas secundárias. ${ }^{11}$

Como vimos acima, um desequilíbrio na resposta imune inata - a limitada produção de IFN I/III com uma robusta produção de quimiocinas/citocinas pró-inflamatórias - é possivelmente um dos fatores iniciais (i.e. da imunidade inata) para promover a proliferação vírica e uma desregulação imune. No entanto, outros componentes da resposta imune adquirida subsequente (linfócitos B e seus atcs, linfócitos T e suas citocinas) poderão contribuir para ampliar e perpetuar essa desregulação imune. Veremos, de seguida, alguns dos mecanismos que têm sido adicionalmente propostos na imunopatologia da COVID-19.

\section{PRODUÇÃO EXCESSIVA DE CITOCINAS}

Alguns vírus são citopáticos, entre os quais os SARS-CoV, i.e. induzem lesão e morte celular nos tecidos infetados por piroptose. ${ }^{41}$ Esta é um tipo de morte celular programada associada a inflamação e exsudação vascular, que contrasta com a apoptose (morte celular não inflamatória) que ocorre na citotoxicidade dependente de mecanismos imunes (células NK ou T citotóxicas). De facto, uma das citocinas pró-inflamatórias caracteristicamente libertada no processo de piroptose, a IL-1 $\beta$, foi recentemente descrita como elevada na infeção pelo SARSCoV-2. ${ }^{10}$ As componentes libertadas pela lise das células epiteliais respiratórias - ATP, ADN, etc. - podem então ser detetadas como DAMPs (damage-associated molecular patterns) pelos PRRs de outras células epiteliais e macrófagos alveolares, resultando na sua ativação. Numa situação fisiológica, essa resposta no pulmão mais profundo seria tendencial à eliminação dos microrganismos, pela atividade de depuração dos macrófagos alveolares. No entanto, em alguns doentes e decorrente p.ex. de uma carga vírica elevada ${ }^{42}$ e/ou do risco imunogenético individual, pode ocorrer uma resposta imune disfuncional, resultando numa hiperinflamação pulmonar difusa e/ou sistémica, desencadeada por uma "tempestade" de citocinas - i.e. uma síndrome de produção excessiva de citocinas (40) ou síndrome de ativação macrofágica. ${ }^{43}$ De facto, observou-se, em doentes graves internados em $\cup C l$ (10), níveis plasmáticos elevados de um conjunto alargado de citocinas como a IL-2, IL-7, IL-10, GCSF (granulocyte colony-stimulating factor), CXCL-10 (IP-10), 
CCL2 (MCP1), CCL-3 (macrophage inflammatory protein $1 \alpha /$ MIP1 $\alpha$ ) e TNF $\alpha$ (tumour necrosis factor). Nesses doentes, os níveis circulantes de IL-6 parecem relacionar-se com o prognóstico, ${ }^{44,11}$ bem como a expressão de marcadores celulares que apontam para ativação de monócitos pró-inflamatórios, quer no pulmão ${ }^{36}$ quer em circulação. ${ }^{45}$ Alguns estudos experimentais, utilizando linhas celulares ou tecido pulmonar ex-vivo, ${ }^{28,46}$ corroboram a indução pelo SARS-CoV-2 de quimiocinas envolvidas no recrutamento e ativação de monócitos-macrófagos, tal como a CCL2 (MCP-1), CCL8 (MCP-2) e CXLC10 (IP10), sugerindo o seu envolvimento na génese da síndrome de libertação citocínica / ativação macrofágica. ${ }^{43,42}$ Adicionalmente, estes dados experimentais ${ }^{28}$ apontam também para um envolvimento dos neutrófilos na COVID-19, dada a indução de outras quimiocinas que recrutam neutrófilos - CXCL2 (GRO『) e CXCL8 (IL-8), observação consistente com a neutrofilia periférica característica destes doentes, que pode ter também significado prognóstico. ${ }^{34,35}$

Para além de um envolvimento local, pulmonar, desta resposta hiperinflamatória, a síndrome de libertação de citocinas tem também efeitos sistémicos. Níveis elevados destas citocinas pró-inflamatórias (IL-1/TNF/IL-6) associam-se a choque e falência multiorgânica, podendo resultar lesão miocárdica, hipotensão e choque, o que tem levado a propor a terminologia de síndrome de "sépsis vírica"39 para o compromisso sistémico observado na COVID-19 grave. Este pode envolver, nalguns casos, hemorragia renal focal, hepatomegalia e hepatite, atrofia esplénica, necrose dos gânglios mediastínicos e mesmo edema cerebral / degenerescência neuronal. Sabe-se que o SARS-CoV-2 pode atingir órgãos que não o pulmão, sobretudo se coexprimem ACE-2 e as protéases a ela associadas, ${ }^{47,48}$ mas não é claro ainda o contributo relativo do vírus e/ou da resposta citocínica do hospedeiro na falência multiorgânica de alguns casos. ${ }^{39}$

\section{PRODUÇÃO DE ANTICORPOS}

Os mecanismos exatos por detrás da progressão da ARDS e produção sistémica da IL-6 na COVID-19 não são ainda claros. Uma observação que tem intrigado clínicos e investigadores $^{19}$ é a coincidência dos sintomas de insuficiência respiratória e ARDS com o aparecimento dos primeiros sinais de uma resposta imune adaptativa, com a presença no sangue de atcs específicos para o SARS-CoV-2. Curiosamente, os níveis de lgA, IgM e lgG específicas parecem mais elevados (e mais precoces) nos doentes com pior evolução clínica. ${ }^{12,49}$ Apesar de esta observação poder representar uma consequência de uma replicação / carga vírica intensa, tem sido levantada a hipótese de que uma resposta imune adaptativa poderá estar a contribuir para patogenia e gravidade da pneumonia. Esta hipótese também seria corroborada pelas formas clínicas mais ligeiras observadas em doentes com imunodeficiência humoral por agamaglobulinemia, como acima já referido.

Os mecanismos potencialmente envolvidos são diversos e poderão relacionar-se com a formação de complexos imunes (ICs) patogénicos. ${ }^{50}$ Os doentes com COVID-19 desenvolvem rapidamente atcs IgA específicos que, formando ICs locais, podem causar inflamação e microtrombose. ${ }^{50,51}$ ICs IgM e IgG podem também originar inflamação e coagulação intravascular, através da ativação do complemento, ${ }^{52}$ e a ligação de ICs vírus-atcs aos recetores Fc dos macrófagos alveolares poderão também ativá-los, com produção de CXCL8 (IL-8) e CCL2 (MCP-1), contribuindo para a inflamação local. ${ }^{53}$ Por outro lado, atcs IgG não neutralizantes para o SARS-CoV-2 poderão favorecer a potenciação da infeção, uma vez que não as neutralizando poderão facilitar a fusão das partículas víricas à membrana das células - a potenciação vírica dependente de atcs. ${ }^{54,55}$ Uma outra observação intrigante é de que algumas sequências do SARS-CoV-2, tal como acontece com outros vírus, podem ter semelhança com constituintes do hospedeiro (por exemplo, proteínas relacionadas ao surfactante pulmonar), potencialmente favorecendo uma reatividade cruzada imunológica e que poderá estar na génese de autoimunidade. ${ }^{56}$ Todos estes dados poderão ser muito informativos para o desenvolvimento e segurança de uma futura vacina.

\section{PARTICIPAÇÃO DE LINFÓCITOS T}

$\mathrm{Na}$ defesa antivírica, a resposta dos linfócitos T é um dos elementos fundamentais da imunidade adaptativa. ${ }^{41}$ As células T CD8+ são importantes pela citotoxicidade específica para as células infetadas (que apresentam peptídeos de origem vírica nas moléculas do MHC classe I da membrana celular); os linfócitos T CD4+ são cruciais para ativar os T CD8+ e os linfócitos $B$, produzindo também citocinas que potenciam recrutamento de células imunocompetentes.

Na resposta ao SARS-CoV-2, foram descritos linfócitos T ativados em circulação aos 7 dias após o início dos sintomas de COVID-19, num doente com infeção moderada e que recuperou totalmente, acompanhando a deteção de linfócitos B ativados e de atcs específicos em circulação. ${ }^{57}$ Já no primeiro estudo de autópsia de um doente com ARDS, ${ }^{38}$ foi descrita redução substancial de linfócitos T em circulação, quer CD4+ quer CD8+, mas com fenótipos de hiperativação (Th17 e de citotoxicidade). Outras séries posteriores, ${ }^{53}$ mais alargadas, referem que os casos mais graves apresentam uma significativa e sustentada redução do número de linfócitos T circulantes, especialmente das células T CD8+, a par de um aumento do número de neutrófilos, sendo que a razão neutrófilos/linfócitos T CD8+ se revelou um fator de prognóstico para a severidade da COVID-19. Essa linfopenia periférica persistente relacionase com a gravidade da COVID-19 e com maior risco de desenvolverem infeções bacterianas secundárias, ${ }^{11}$ sendo uma das características que suporta um estádio de imunossupressão que se seguirá à fase de hiperinflamação da COVID-19.39,40 As causas dessa linfopenia periférica não estão identificadas, sendo que um efeito citopático do vírus não parece provável, dado os linfócitos T não exprimirem ACE-2. Tem sido sugerido 
IMUNOLOGIA DA DOENÇA POR CORONAVÍRUS-19 (COVID-19): UMA PERSPETIVA PARA O CLÍNICO, NOS PRIMEIROS 4 MESES DA EMERGÊNCIA DO SARS-COV-2

Tabela 1: Intervenções terapêuticas com base na resposta imunológica e imunopatologia da COVID-19

\begin{tabular}{l|l|l|}
\hline \multicolumn{1}{|c|}{ TIPO } & MECANISMO / AÇÕES & REFERENCIAS \\
\hline Citocinas & Inibição da replicação viral; uso isolado ou em combinação com antivirais & $66 ; 67 ; 41 ; 68$ \\
\hline $\begin{array}{l}\text { IFN tipo I ( } \alpha \text { ou } \beta \text { ) } \\
\text { IFN tipo III (peg-IFN- } \lambda 1)\end{array}$ & $\begin{array}{l}\text { Indução da proliferação de linfócitos T naive e de memória (CD4 e CD8); } \\
\text { recuperação da linfopenia (?). Sem estudos na COVID-19 }\end{array}$ & $69 ; 70$ \\
\hline IL-7 recombinante & & \\
\hline Anti-citocinas & &
\end{tabular}

Inibidores do recetor da IL-6 (Tocilizumab/

Sarilumab)

Inibição da IL-6 ou recetores (um dos principais mediadores da tempestade

$71 ; 72 ; 73 ; 74 ; 75$

Inibidores da IL-6 (Siltuximab)

de citocinas)

Clazakinumab/Sirukumab)

Inibidores da IL-1

Anakinra (recetor recombinante de IL-1)

Inibição da citocina pró-inflamatória IL-1 beta (síndrome de ativação macrofágica)

$76 ; 77$

Canakinumab (MoAb anti IL-1beta)

\begin{tabular}{l|l}
\hline $\begin{array}{l}\text { Inibidores da IL-18 } \\
\text { Tadekinig alfa }\end{array}$ & Inibição da IL-18 (síndrome de ativação macrofágica, doença auto-infla- \\
(proteína de ligação à IL-18 recombinante) & matória)
\end{tabular}

\begin{tabular}{l|l|l}
\hline Inibidores de IFN $\gamma$ & Inibição do IFN $\gamma$ (síndrome de ativação macrofágica) & $79 ; 80$ \\
\hline Emapalumab (MoAb anti IFN $\gamma$ ) & \\
\hline
\end{tabular}

\begin{tabular}{l|l}
\hline Inibidores do TNF $\alpha$ & \\
Etanercept (proteína quimérica do & Inibição do TNF $\alpha$ com consequente diminuição de IL-1, IL-6, moléculas de \\
receptor do TNF) & adesão e tráfego de leucócitos
\end{tabular}

Adalimumab (MoAb anti TNF)

\section{Inibidores da via JAK/STAT}

\begin{tabular}{|l|l|l|l|}
\hline $\begin{array}{l}\text { Tofacitinib } \\
\text { Baricitinib } \\
\text { Ruxolitinib }\end{array}$ & Inibição da sinalização para múltiplas vias da ativação de citocinas. Risco & $83 ; 84$ \\
aumentado de tromboembolismo e diminuição de IL-7 e IFN tipo I
\end{tabular}

\begin{tabular}{|c|c|c|}
\hline \multicolumn{3}{|l|}{ Imunoglobulinas } \\
\hline $\begin{array}{l}\text { Plasma convalescente e Imunoglobulina } \\
\text { hiperimune }\end{array}$ & $\begin{array}{l}\text { Neutralização do vírus; controlo da hipercoagulabilidade; modulação da liber- } \\
\text { tação descontrolada de citocinas }\end{array}$ & $85 ; 86 ; 87 ; 15$ \\
\hline Imunoglobulina endovenosa & Imunomodulação (síndrome de ativação macrofágica; sépsis) & 88 \\
\hline \multicolumn{3}{|l|}{ Outros } \\
\hline $\begin{array}{l}\text { Bevacizumab (MoAb anti VEGF) } \\
\text { Eculizumab (MoAb anti C5) }\end{array}$ & $\begin{array}{l}\text { Anti VEGF } \\
\text { Inibidor do complemento terminal }\end{array}$ & $89 ; 19$ \\
\hline
\end{tabular}

NC5 - fator 5 do Complemento; MoAb - monoclonal antibody; VEGF - vascular endothelial growth factor

Adaptado de: ClinicalTrials.gov https://clinicaltrials.gov/ct2/home; WHO, Landscape of COVID-19 candidate vaccines - 20 April 2020 (draft) (https://www.who.int/blueprint/priority-diseases/key-action/novel-coronavirus-landscape-ncov.pdf)

estar relacionada com o recrutamento intenso aos órgãos infetados - aparelhos respiratório e gastrointestinal, ${ }^{41}$ ou com mecanismos de apoptose induzida por ativação via Fas/Ligando do Fas, ou apoptose relacionada com o TNF.39,58

\section{OUTROS MECANISMOS PATOGÉNICOS}

Coagulação intravascular - alguns estudos histopatológicos post-mortem ${ }^{59}$ de tecido pulmonar descrevem, a par de lesão alveolar difusa, congestão pulmonar, trombose e oclusão microvascular. Parâmetros de coagulação alterados e elevação do D-dímero são biomarcadores desta perturbação e de um pior prognóstico. ${ }^{60}$

Sistema renina-angiotensina e ACE-2 - tal como para o
SARS-CoV-1, o recetor celular para o SARS-CoV-2 é a ACE-2, expressa no epitélio, macrófagos e endotélio vascular do pulmão. A infeção pelos coronavírus da SARS induz uma redução da expressão de ACE-2 no pulmão. ${ }^{41}$ A ACE-2 participa na regulação do sistema renina-angiotensina, podendo a sua redução pós infeção acarretar disfunção deste sistema, influenciando a pressão arterial, equilíbrio hidroeletrolítico e potenciando a permeabilidade vascular pulmonar e inflamação. ${ }^{61}$

\section{Conclusão}

Nesta revisão, visitámos vários dos mecanismos envolvidos na resposta imunológica ao SARS-CoV-2, conhecimentos 
que são o fruto de uma resposta global de muitos investigadores e clínicos em todo o Mundo, num esforço comum para melhor entender a patogenia da COVID-19. Muitos dos dados já obtidos nestes primeiros 4 meses permitem concluir que tão importante como atingir o vírus e os seus mecanismos de replicação, será o controlo da resposta inflamatória e da desregulação imunitária subjacentes à infeção. Assim, estão já propostas, ou em ensaio, múltiplas intervenções terapêuticas com base nos conhecimentos da resposta imunológica e imunopatologia da COVID-19 (Tabela 1). Paralelamente, o melhor reconhecimento da disfunção imunitária associada às formas clinicamente mais graves e à imunopatologia da COVID-19, poderá melhor acautelar os efeitos adversos e indesejáveis no desenvolvimento e avaliação de uma nova vacina (Tabela 2) e das suas múltiplas estratégias. ${ }^{62-65}$

Finalmente, lembrar que colocamos aqui várias hipóteses, fruto do acompanhamento e revisão da literatura médica e científica muito recente que, como toda a ciência, necessita de ser replicada por diferentes grupos, para consolidação dos seus resultados e conclusões. Muitas das hipóteses aqui expostas foram alicerçadas numa discussão alargada com investigadores, académicos e clínicos que acompanham e enfrentam esta nova realidade da COVID-19, e serão incompletas ou mesmo, num futuro próximo, comprovadamente erradas. Em todo o caso, esperamos que esta revisão se possa alinhar com a quarta e última das áreas estratégicas definidas pela Organização Mundial de Saúde ${ }^{3}$ para controlar esta pandemia: inovar e aprender.

\section{Agradecimento}

Os autores agradecem a todos os colegas que contribuíram com a troca de experiências, informações e discussão, para a organização desta revisão, nomeadamente Ana Margarida Pereira, André Moreira, Andrea Leonardi, Bernardo Sousa Pinto, Cristina Lopes, Diana Silva, Jean-Luc Fauquert, João Fonseca, Jorge Palmares, José Artur Paiva, Luís Araújo, Osvaldo Correia, Paolo Matricardi e Tiago Rama.

\section{Responsabilidades Éticas}

Conflitos de Interesse: Os autores declaram a inexistência de conflitos de interesse na realização do presente trabalho.

Fontes de Financiamento: Não existiram fontes externas de financiamento para a realização deste artigo.

Proveniência e Revisão por Pares: Comissionado; sem revisão externa por pares.

\section{Ethical Disclosures}

Conflicts of interest: The authors have no conflicts of interest to declare. Financing Support: This work has not received any contribution, grant or scholarship.

Confidentiality of Data: The authors declare that they have followed the protocols of their work center on the publication of data from patients.

Provenance and Peer Review: Commissioned; without externally peer reviewed.

(c) Autor (es) (ou seu (s) empregador (es)) 2019. Reutilização permitida de acordo com CC BY-NC. Nenhuma reutilização comercial.

(C) Author(s) (or their employer(s)) 2019. Re-use permitted under CC BYNC. No commercial re-use.

Tabela 2: Vantagens e desvantagens das vacinas candidatas para Covid-19 em fase clínica

\begin{tabular}{|c|c|c|c|}
\hline Estratégia & Vantagens & Desvantagens & Vacinas candidatas \\
\hline $\begin{array}{l}\text { Vacinas de vírus } \\
\text { inativados }\end{array}$ & $\begin{array}{l}\text { Fácil produção; segura; } \\
\text { altos títulos de acs neu- } \\
\text { tralizantes }\end{array}$ & $\begin{array}{l}\text { Potencialmente inapropriada } \\
\text { para indivíduos imunossu- } \\
\text { primidos }\end{array}$ & $\begin{array}{l}\text { Vacina de vírus inativado, PiCoVacc (Sinovac, } \\
\text { Fase I, NCT04352608) } \\
\text { Vacina de vírus inativado, (Wuhan Institute of Bio- } \\
\text { logical Products, Fase I, ChiCTR2000031809) }\end{array}$ \\
\hline $\begin{array}{l}\text { Vacinas de vírus } \\
\text { atenuados }\end{array}$ & $\begin{array}{l}\text { Rápido desenvolvimento; } \\
\text { boa indutora de respos- } \\
\text { tas imunes }\end{array}$ & $\begin{array}{l}\text { Reversão fenotípica ou } \\
\text { genotípica possível; pode } \\
\text { provocar doença; contra-in- } \\
\text { dicada em imunossuprimidos }\end{array}$ & $\begin{array}{l}\text { Vacina de vírus vivo atenuado (Codagenix/ Serum } \\
\text { Institute of India, Fase Pré-clínica, sem registo) }\end{array}$ \\
\hline $\begin{array}{l}\text { Vacinas de } \\
\text { subunidades } \\
\text { virais (proteínas } \\
\text { recombinantes) }\end{array}$ & $\begin{array}{l}\text { Muito segura; produção } \\
\text { consistente; pode induzir } \\
\text { respostas imunes celular } \\
\text { e humoral; altos títulos de } \\
\text { acs neutralizantes. }\end{array}$ & $\begin{array}{l}\text { Alto custo; baixa imunogeni- } \\
\text { cidade; requer doses repeti- } \\
\text { das e uso de adjuvantes }\end{array}$ & $\begin{array}{l}\text { DCs ou aAPCs modificadas com vetor lentiviral } \\
\text { expressando genes sintéticos baseados em } \\
\text { domínios de ptns virais selecionadas (Shen- } \\
\text { zhen Geno-Immune Medical Institute, Fase I, } \\
\text { NCT04276896 e NCT04299724) }\end{array}$ \\
\hline Vacinas de ADN & $\begin{array}{l}\text { Desenho simples; muito } \\
\text { segura; altos títulos de } \\
\text { acs neutralizantes }\end{array}$ & $\begin{array}{l}\text { Resposta imune em huma- } \\
\text { nos inferior à observada em } \\
\text { animais; doses repetidas po- } \\
\text { dem cursar com toxicidade }\end{array}$ & $\begin{array}{l}\text { Plasmídeo de DNA codificador de ptn S entregue } \\
\text { por eletroporação (Inovio Pharmaceuticals, Fase I, } \\
\text { NCT04336410) }\end{array}$ \\
\hline Vacinas de ARNm & $\begin{array}{l}\text { Desenho simples; } \\
\text { induz respostas imunes } \\
\text { potentes }\end{array}$ & $\begin{array}{l}\text { Altamente instável sob } \\
\text { condições fisiológicas }\end{array}$ & $\begin{array}{l}\text { Vacina de RNAm encapsulada em nanopartícula } \\
\text { de lipido codificadora de ptn S-1273 (Modernal } \\
\text { NIAID - Fase I, NCTO4283461) }\end{array}$ \\
\hline
\end{tabular}

aAPCs - artificial antigen-presenting cell; acs - anticorpos; DC - dendritic cell; ptn = proteína 
Correspondence/Correspondência: Luís Delgado

Idelgado@med.up.pt

Serviço de Imunologia Básica e Clínica, Departamento de Patologia,

Faculdade de Medicina, Universidade do Porto, Portugal.

Alameda Prof. Hernâni Monteiro, 4200-319 Porto

Received/Recebido: 14/05/2020

Accepted/Aceite: 14/05/2020

Publicado / Published: 19 de Maio de 2020

\section{REFERÊNCIAS}

1. Tyrrell DAJ, Bynoe ML. Cultivation of a novel type of common-cold virus in organ cultures. Br Med J. 1965;1:1467-70. 10.1136/bmj.1.5448.1467

2. Heymann DL, Shindo N; WHO Scientific and Technical Advisory Group for Infectious Hazards. COVID-19: what is next for public health? Lancet. 2020;395:542-5. doi:10.1016/S0140-6736(20)30374-3

3. WHO Director-General; World Health Organization opening remarks at the media briefing on COVID-19 - [accessed 11 March 2020 WHO https:// www.who.int/dg/ speeches/detail/who- director- general- s- openingremarks- at- the- media- briefing- on- covid-19--11- march-2020 .

4. Zagury-Orly I, Schwartzstein RM. Covid-19 - A Reminder to Reason [published online ahead of print, 2020 Apr 28]. N Engl J Med. 2020;10.1056/ NEJMp2009405. doi:10.1056/ NEJMp2009405

5. Lu X, Zhang L, Du H, Zhang J, Li Y, Qu J, et al. SARS-CoV-2 Infection in Children. N Engl J Med. 2020;382:1663-5. doi:10.1056/NEJMc2005073

6. Hoehl S, Rabenau H, Berger A, Kortenbusch M, Cinatl J, Bojkova D, et al. Evidence of SARS-CoV-2 Infection in Returning Travelers from Wuhan, China. N Engl J Med. 2020;382:1278-80. doi:10.1056/NEJMc2001899

7. Grasselli G, Zangrillo A, Zanella A, Antonelli M, Cabrini L, Castelli A, et al. Baseline Characteristics and Outcomes of 1591 Patients Infected With SARS-CoV-2 Admitted to ICUs of the Lombardy Region, Italy. JAMA. 2020;323:1574-81. doi:10.1001/jama.2020.5394

8. Lippi G, Mattiuzzi C, Sanchis-Gomar F, Henry BM. Clinical and demographic characteristics of patients dying from COVID-19 in Italy versus China. J Med Virol. 2020 (in press). doi:10.1002/jmv.25860

9. Zhu N, Zhang D, Wang W, Li X, Yang B, Song J, et al. A Novel Coronavirus from Patients with Pneumonia in China, 2019. N Engl J Med. 2020;382:72733. doi:10.1056/NEJMoa2001017

10. Huang C, Wang Y, Li X, Ren L, Zhao J, Hu Y, et al. Clinical features of patients infected with 2019 novel coronavirus in Wuhan, China [published correction appears in Lancet. 2020 Jan 30]. Lancet. 2020;395:497-506. doi:10.1016/S0140-6736(20)30183-5

11. Zhou F, Yu T, Du R, Fan G, Liu Y, Liu Z, et al. Clinical course and risk factors for mortality of adult inpatients with COVID-19 in Wuhan, China: a retrospective cohort study [published correction appears in Lancet. 2020 Mar 28;395(10229):1038] [published correction appears in Lancet. $2020 \mathrm{Mar}$ 28;395(10229):1038]. Lancet. 2020;395(10229):1054-1062. doi:10.1016/ S0140-6736(20)30566-3

12. Okba NMA, Müller MA, Li W, Wang C, Geurtsvan Kessel CH, Corman VM, et al. Severe Acute Respiratory Syndrome Coronavirus 2-Specific Antibody Responses in Coronavirus Disease 2019 Patients. Emerg Infect Dis. 2020;26:10.3201/eid2607.200841. doi:10.3201/eid2607.200841

13. Guo L, Ren L, Yang S, Xiao M, Chang D, Yang F, et al. Profiling Early Humoral Response to Diagnose Novel Coronavirus Disease (COVID-19). Clin Infect Dis. 2020 (in press). doi:10.1093/cid/ciaa310

14. Zhao J, Yuan Q, Wang H, Liu W, Liao X, Su Y, et al. Antibody responses to SARS-CoV-2 in patients of novel coronavirus disease 2019. Clin Infect Dis. 2020 (in press). doi:10.1093/cid/ciaa344

15. Rubin R. Testing an Old Therapy Against a New Disease: Convalescent Plasma for COVID-19 JAMA. 2020 (in press).doi:10.1001/jama.2020.7456

16. Wu F, Wang A, Liu M, Wang Q, Chen J, Xia S et al. Neutralizing antibody responses to SARS-CoV-2 in a COVID-19 recovered patient cohort and their implications. medRxiv 2020.03.30.20047365; doi: https://doi. org/10.1101/2020.03.30.20047365
17. Soresina A, Moratto D, Chiarini M, Paolillo C, Baresi G, Focà E, et al. Two $X$-linked agammaglobulinemia patients develop pneumonia as COVID19 manifestation but recover.Pediatr Allergy Immunol. 2020 (in press). doi:10.1111/pai.13263

18. Quinti I, Lougaris V, Milito C, Cinetto F, Pecoraro A, Mezzaroma I, et al. A possible role for B cells in COVID-19?: Lesson from patients with Agammaglobulinemia. J Allergy Clin Immunol. 2020 (in press). doi:10.1016/j. jaci.2020.04.013

19. Matricardi PM, Dal Negro RW, Nisini R. The first, holistic immunological model of COVID-19: implications for prevention, diagnosis, and public health measures. Pediatr Allergy Immunol. 2020 (in press). doi:10.1111/ pai. 13271

20. Watanabe Y, Allen JD, Wrapp D, McLellan JS, Crispin M. Site-specific analysis of the SARSCoV- 2 glycan shield. bioRxiv preprint 2020. [e-pub] doi. org/10.1101/2020.03.26.010322

21. Cheng $\mathrm{Y}$, Cheng $\mathrm{G}$, Chui $\mathrm{CH}$, Lau FY, Chan PK, Ng MH, et al. ABO blood group and susceptibility to severe acute respiratory syndrome [published correction appears in JAMA. 2005;294:794. Cheng, Yufeng [corrected to Cheng, Yunfeng]. JAMA. 2005;293:1450-1. doi:10.1001/ jama.293.12.1450-c

22. Zhao J, Yang Y, Huang H, Li D, Gu D, Lu X, et al. Relationship between the ABO Blood Group and the COVID-19 Susceptibility. medRxiv PREPRINT. 2020. [e-pub] doi.org/10.1101/ 2020.03.11.20031096.

23. Zhou Y, Lu K, Pfefferle S, Bertram S, Glowacka I, Drosten C, et al. A single asparagine-linked glycosylation site of the severe acute respiratory syndrome coronavirus spike glycoprotein facilitates inhibition by mannose-binding lectin through multiple mechanisms. J Virol. 2010;84:8753-64. doi:10.1128/ JVI.00554-10

24. Zhang H, Zhou G, Zhi L, Yang H, Zhai Y, Dong X, et al. Association between mannose-binding lectin gene polymorphisms and susceptibility to severe acute respiratory syndrome coronavirus infection. J Infect Dis. 2005;192:1355-61. doi:10.1086/491479

25. Tomaiuolo R, Ruocco A, Salapete C, Carru C, Baggio G, Franceschi C, et al. Activity of mannose-binding lectin in centenarians. Aging Cell. 2012;11:394400. doi:10.1111/j.1474-9726.2012.00793.x

26. Andreakos E, Zanoni I, Galani IE. Lambda interferons come to light: dual function cytokines mediating antiviral immunity and damage control. Curr Opin Immunol. 2019;56:67-75. doi:10.1016/j.coi.2018.10.007

27. Boudewijns R, Thibaut HJ, et al. STAT2 signaling as double-edged sword restricting viral dissemination but driving severe pneumonia in SARS-CoV-2 infected hamsters. bioRxiv preprint doi: https://doi. org/10.1101/2020.04.23.056838.

28. Blanco-Melo D, Nilsson-Payant B, Liu W, Uhl S, Hoagland D et al Imbalanced host response to SARS-CoV-2 drives development of COVID-19. Cell. 2020 (in press) doi: 10.1016/j.cell.2020.04.026.

29. Deng X, Chen Y, Mielech AM, et al. Structure-Guided Mutagenesis Alters Deubiquitinating Activity and Attenuates Pathogenesis of a Murine Coronavirus. J Virol. 2020 (in press). doi:10.1128/JVI.01734-19

30. García-Sastre A. Ten Strategies of Interferon Evasion by Viruses. Cell Host Microbe. 2017;22:176-84. doi:10.1016/j.chom.2017.07.012

31. Galani IE, Triantafyllia V, Eleminiadou EE, Koltsida O, Stavropoulos A, Manioudaki M, et al. Interferon- $\lambda$ Mediates Non-redundant Front-Line Antiviral Protection against Influenza Virus Infection without Compromising Host Fitness. Immunity. 2017;46:875-90.e6. doi:10.1016/j.immuni.2017.04.025

32. Klinkhammer J, Schnepf D, Ye L, Schwaderlapp M, Gad HH, Hartmann $\mathrm{R}$, et al. IFN- $\lambda$ prevents influenza virus spread from the upper airways to the lungs and limits virus transmission. Elife. 2018;7:e33354. doi:10.7554/ elife.33354

33. Ye L, Schnepf D, Staeheli P. Interferon- $\lambda$ orchestrates innate and adaptive mucosal immune responses. Nat Rev Immunol. 2019;19:614-25. doi:10.1038/s41577-019-0182-z

34. Chen N, Zhou M, Dong $X$, et al. Epidemiological and clinical characteristics of 99 cases of 2019 novel coronavirus pneumonia in Wuhan, China: a descriptive study. Lancet. 2020;395:507-13. doi:10.1016/S01406736(20)30211-7

35. Qin C, Zhou L, Hu Z, Zhang S, Yang S, Tao Y, et al. Dysregulation of immune response in patients with COVID-19 in Wuhan, China. Clin Infect Dis. 2020 
(in press). doi: $10.1093 / \mathrm{cid} / \mathrm{ciaa248}$

36. Liao M, Liu Y, Yuan J, Wen Y, Xu G, Zhao J, et al. The landscape of lung bronchoalveolar immune cells in COVID-19 revealed by single-cell RNA sequencing. medRxiv, 2020. doi:10.1101/2020.02.23.20026690

37. Wong CK, Lam CW, Wu AK, Ip WK, Lee NL, Chan IH, et al. Plasma inflammatory cytokines and chemokines in severe acute respiratory syndrome. Clin Exp Immunol. 2004;136:95-103. doi:10.1111/j.1365-2249.2004.02415.x

38. Xu Z, Shi L, Wang Y, Zhang J, Huang L, Zhang $C$ et al. Pathological findings of COVID-19 associated with acute respiratory distress syndromeLancet Respir Med. 2020;8:420-2. doi:10.1016/S2213-2600(20)30076-X

39. Li H, Liu L, Zhang D, Xu J, Dai H, Tang N, et al. SARS-CoV-2 and vira sepsis: observations and hypotheses. Lancet. 2020 (in press). doi:10.1016/ S0140-6736(20)30920-X

40. Mehta P, McAuley DF, Brown M, Sanchez E, Tattersall RS, Manson JJ. COVID-19: consider cytokine storm syndromes and immunosuppression. Lancet. 2020;395:1033-4. doi:10.1016/S0140-6736(20)30628-0

41. Tay MZ, Poh CM, Rénia L, MacAry PA, Ng LFP. The trinity of COVID-19: immunity, inflammation and intervention. Nat Rev Immunol. 2020;1-12. doi:10.1038/s41577-020-0311-8

42. Shi Y, Wang Y, Shao C, Huang J, Gan J, Huang X, et al. COVID-19 infection: the perspectives on immune responses. Cell Death Differ. 2020;27:1451-4. doi:10.1038/s41418-020-0530-3

43. Shoenfeld Y. Corona (COVID-19) time musings: Our involvement in COVID19 pathogenesis, diagnosis, treatment and vaccine planning. Autoimmun Rev. 2020;19:102538. doi:10.1016/j.autrev.2020.102538

44. Herold T, Jurinovic V, Arnreich C, Hellmuth JC, von Bergwelt- Baildon M Klein M, Weinberger T. Level of IL-6 predicts respiratory failure in hospitalized symptomatic COVID-19 patients. Pre-print. doi: https://doi.org/ 10.1101/2020.04.01.20047381

45. Zhou Y, Fu B, Zheng X, Wang D, Zhao C, Qi Y, et al. Pathogenic T cells and inflammatory monocytes incite inflammatory storm in severe COVID-19 patients. Natl Sci Rev. 2020 (in press). doi:10.1093/nsr/nwaa041.

46. Chu $\mathrm{H}$, Chan JF, Wang $\mathrm{Y}$, et al. Comparative replication and immune activation profiles of SARS-CoV-2 and SARS-CoV in human lungs: an ex vivo study with implications for the pathogenesis of COVID-19. Clin Infect Dis. 2020 (in press). doi:10.1093/cid/ciaa410

47. Varga Z, Flammer AJ, Steiger P, Haberecker M, Andermatt R, Zinkernage AS, et al. Endothelial cell infection and endothelitis in COVID-19. Lancet. 2020;395:1417-8. doi:10.1016/S0140-6736(20)30937-5

48. Muus C, Luecken MD, Eraslan G, Waghray A, Heimberg G, Sikemma L, et al. Integrated analyses of single-cell atlases reveal age, gender, and smoking status associations with cell type-specific expression of mediators of SARS-CoV-2 viral entry and highlights inflammatory programs in putative target cells. bioRxiv 2020.04.19.049254; doi: https://doi. org/10.1101/2020.04.19.049254

49. Padoan A, Cosma C, Sciacovelli L, Faggian D, Plebani M. Analytical performances of a chemiluminescence immunoassay for SARS-CoV-2 IgM/lgG and antibody kinetics. Clin Chem Lab Med. 2020 (in press). doi:10.1515/ cclm-2020-0443

50. Monsalvo AC, Batalle JP, Lopez MF, Krause JC, Klemenc J, Hernandez JZ, et al. Severe pandemic 2009 H1N1 influenza disease due to pathogenic immune complexes. Nat Med. 2011;17:195-9. doi:10.1038/nm.2262

51. Dahlke C, Heidepriem J, Kobbe R, Santer R, Koch T, Fathi A, et al Distinct early IgA profile may determine severity of COVID-19 symptoms: an immunological case series. medRxiv preprint doi: https://doi. org/10.1101/2020.04.14.20059733

52. Ciceri F, Beretta L, Scandroglio AM, Colombo S, Landoni G, Ruggeri A, et al. Microvascular COVID-19 lung vessels obstructive thromboinflammatory syndrome (MicroCLOTS): an atypical acute respiratory distress syndrome working hypothesis. Crit Care Resusc. 2020 (in press).

53. Liu L, Wei Q, Lin Q, Fang J, Wang H, Kwok H, et al. Anti-spike IgG causes severe acute lung injury by skewing macrophage responses during acute SARS-CoV infection. JCl Insight. 2019;4:e123158. doi:10.1172/jci.insight.123158

54. Walls AC, Xiong X, Park YJ, Tortorici MA, Snijder J, Quispe J, et al. Unexpected Receptor Functional Mimicry Elucidates Activation of Coronavirus Fusion. Cell. 2019;176:1026-1039.e15. doi:10.1016/j.cell.2018.12.028
55. Wan Y, Shang J, Sun S, Tai W, Chen J, Geng Q, et al. Molecular Mechanism for Antibody-Dependent Enhancement of Coronavirus Entry. J Virol. 2020;94:e02015-19. doi:10.1128/JVI.02015-19

56. Kanduc D, Shoenfeld Y. On the molecular determinants of the SARS-CoV-2 attack. Clin Immunol. 2020;215:108426. doi:10.1016/j.clim.2020.108426

57. Thevarajan I, Nguyen THO, Koutsakos M, Druce J, Caly L, van de Sandt $\mathrm{CE}$, et al. Breadth of concomitant immune responses prior to patient recovery: a case report of non-severe COVID-19. Nat Med. 2020;26:453-5. doi:10.1038/s41591-020-0819-2

58. Peteranderl C, Herold S. The Impact of the Interferon/TNF-Related Apoptosis-Inducing Ligand Signaling Axis on Disease Progression in Respiratory Viral Infection and Beyond. Front Immunol. 2017;8:313. doi:10.3389/ fimmu.2017.00313

59. Tian S, Xiong Y, Liu H, Niu L, Guo J, Liao M, Xiao SY. Pathological study of the 2019 novel coronavirus disease (COVID-19) through postmortem core biopsies. Mod Pathol. 2020;1-8. doi:10.1038/s41379-020-0536-x

60. Wang J, Hajizadeh N, Moore EE, Mclntyre RC, Moore PK, Veress LA, et al. Tissue Plasminogen Activator (tPA) Treatment for COVID-19 Associated Acute Respiratory Distress Syndrome (ARDS): A Case Series. J Thromb Haemost. 2020 (in press). doi:10.1111/jth.14828

61. Kuba K, Imai Y, Rao S, Gao H, Guo F, Guan B, et al. A crucial role of angiotensin converting enzyme 2 (ACE2) in SARS coronavirus-induced lung injury. Nat Med. 2005;11:875-9. doi:10.1038/nm1267

62. Amanat F, Krammer F. SARS-CoV-2 Vaccines: Status Report. Immunity. 2020;52:583-9. doi:10.1016/j.immuni.2020.03.007

63. Thanh Le T, Andreadakis Z, Kumar A, Roman RG, Tollefsen S, Saville M, et al. The COVID-19 vaccine development landscape. Nat Rev Drug Discov. 2020;19:305-6. doi:10.1038/d41573-020-00073-5

64. Mahase E. Covid-19: What do we know so far about a vaccine? BMJ. 2020;369:m1679. doi:10.1136/bmj.m1679

65. Shang $W$, Yang Y, Rao Y, Rao X. The outbreak of SARS-CoV-2 pneumonia calls for viral vaccines. NPJ Vaccines. 2020;5:18. doi:10.1038/s41541-0200170-0

66. Zhou Q, Wei X-S, Xiang X, Wang X, Wang Z-H, Chen V, et al. Interferon-a2b treatment for COVID-19. MedRxiv 2020:2020.04.06.20042580.https://doi. org/10.1101/2020.04.06.20042580.

67. Prokunina-Olsson L, Alphonse N, Dickenson RE, Durbin JE, Glenn JS, Hartmann R, et al. COVID-19 and emerging viral infections: The case for interferon lambda. J Exp Med. 2020;217:e20200653. doi:10.1084/jem.20200653

68. Hung IF, Lung K, Tso EY, Liu R, Chung TW, Chu M, et al. Triple combination of interferon beta- $1 \mathrm{~b}$, lopinavir-ritonavir, and ribavirin in the treatment of patients admitted to hospital with COVID-19: an open-label, randomised, phase 2 trial. Lancet. 2020 (in press). doi: 10.1016/S01406736(20)31042-4

69. Francois B, Jeannet R, Daix T, Walton AH, Shotwell MS, Unsinger J, et al. Interleukin-7 restores lymphocytes in septic shock: the IRIS-7 randomized clinical trial. JCI Insight. 2018;3. doi:10.1172/jci.insight.98960

70. Thiébaut R, Jarne A, Routy JP, Sereti I, Fischl M, Ive P, et al. Repeated Cycles of Recombinant Human Interleukin 7 in HIV-Infected Patients With Low CD4 T-Cell Reconstitution on Antiretroviral Therapy: Results of 2 Phase II Multicenter Studies. Clin Infect Dis. 2016;62:1178-85. doi:10.1093/cid/ ciw065

71. Xu X, Han M, Li T, Sun W, Wang D, Fu B, et al. Effective treatment of severe COVID-19 patients with tocilizumab. Proc Natl Acad Sci U S A. 2020(in press). doi:10.1073/pnas.2005615117

72. Luo P, Liu Y, Qiu L, Liu X, Liu D, Li J. Tocilizumab treatment in COVID19: A single center experience. J Med Virol. 2020 (in press). doi:10.1002/ jmv. 25801

73. Gritti G, Raimondi F, Ripamonti D, Riva I, Landi F, Alborghetti L, et al. Use of siltuximab in patients with COVID-19 pneumonia requiring ventilatory support. MedRxiv 2020:2020.04.01.20048561. https://doi.org/10.1101/2020. 04.01 .20048561$.

74. Michot JM, Albiges L, Chaput N, Saada V, Pommeret F, Griscelli F, et al. Tocilizumab, an anti-IL6 receptor antibody, to treat Covid-19-related respiratory failure: a case report. Ann Oncol. 2020 (in press). doi:10.1016/j. annonc.2020.03.300

75. Roumier M, Paule R, Groh M, Vallee A, Ackermann F. Interleukin-6 blockade 
for severe COVID-19. MedRxiv 2020:2020.04.20.20061861. https://doi.or g/10.1101/2020.04.20.20061861.

76. Wampler Muskardin TL. IV anakinra for macrophage activation syndrome may hold lessons for treatment of cytokine storm in the setting of COVID19. ACR Open Rheumatol. 2020 (in press). doi:10.1002/acr2.11140

77. Shakoory B, Carcillo JA, Chatham WW, Amdur RL, Zhao H, Dinarello CA, et al. Interleukin-1 Receptor Blockade Is Associated With Reduced Mortality in Sepsis Patients With Features of Macrophage Activation Syndrome: Reanalysis of a Prior Phase III Trial. Crit Care Med. 2016;44:275-81. doi:10.1097/CCM.0000000000001402

78. Weiss ES, Girard-Guyonvarc'h C, Holzinger D, de Jesus AA, Tariq Z, Picarsic J, et al. Interleukin-18 diagnostically distinguishes and pathogenically promotes human and murine macrophage activation syndrome. Blood. 2018;131:1442-55. doi:10.1182/blood-2017-12-820852

79. Vallurupalli M, Berliner N. Emapalumab for the treatment of relapsed/refractory hemophagocytic lymphohistiocytosis. Blood. 2019;134:1783-6. doi:10.1182/blood.2019002289

80. Lagunas-Rangel FA, Chávez-Valencia V. High IL-6/IFN- $\gamma$ ratio could be associated with severe disease in COVID-19 patients. J Med Virol. 2020 (in press). doi:10.1002/jmv.25900

81. Feldmann M, Maini RN, Woody JN, Holgate ST, Winter G, Rowland M, et al. Trials of anti-tumour necrosis factor therapy for COVID-19 are urgently needed. Lancet. 2020;395:1407-9. doi:10.1016/S0140-6736(20)30858-8

82. McDermott JE, Mitchell HD, Gralinski LE, Eisfeld AJ, Josset L, Bankhead A, et al. The effect of inhibition of PP1 and TNF $\alpha$ signaling on pathogenesis of SARS coronavirus. BMC Syst Biol. 2016;10:93. doi:10.1186/s12918-0160336-6

83. Jamilloux Y, El Jammal T, Vuitton L, Gerfaud-Valentin M, Kerever S, Sève P. JAK inhibitors for the treatment of autoimmune and inflammatory diseases. Autoimmun Rev. 2019;182390. doi:10.1016/j.autrev.2019.102390

84. Favalli EG, Ingegnoli F, De Lucia O, Cincinelli G, Cimaz R, Caporali R. COVID-19 infection and rheumatoid arthritis: Faraway, so close!. Autoimmun Rev. 2020;19:102523. doi:10.1016/j.autrev.2020.102523

85. Duan K, Liu B, Li C, Zhang H, Yu T, Qu J, et al. Effectiveness of convalescent plasma therapy in severe COVID-19 patients. Proc Natl Acad Sci U S A. 2020;117:9490-9496. doi:10.1073/pnas.2004168117

86. Shen C, Wang Z, Zhao F, Yang Y, Li J, Yuan J, et al. Treatment of 5 Critically III Patients With COVID-19 With Convalescent Plasma. JAMA. 2020;323:1582-9. doi:10.1001/jama.2020.4783

87. Langhi DM, Santis GC, Bordin JO. COVID-19 convalescent plasma transfusion. Hematol Transfus Cell Ther. 2020 (in press). doi:10.1016/j. htct.2020.04.003

88. Cao W, Liu X, Bai T, et al. High-Dose Intravenous Immunoglobulin as a Therapeutic Option for Deteriorating Patients With Coronavirus Disease 2019. Open Forum Infect Dis. 2020;7:ofaa102. doi:10.1093/ofid/ofaa102

89. Sanders JM, Monogue ML, Jodlowski TZ, Cutrell JB. Pharmacologic Treatments for Coronavirus Disease 2019 (COVID-19): A Review. JAMA. 2020 (in press). doi:10.1001/jama.2020.6019 